\title{
The Genetic Diversity of Spirometra Tapeworm Isolates From Snakes in The Hunan Province of Central China
}

\section{Tengfang Gong}

Hunan Agricultural University

\section{Fangfang $X u$}

Zhengzhou University

\section{Fen Li}

Hunan Agricultural University

\section{Junlin He}

Hunan Agricultural University

\section{Shuyu Chen}

Hunan Agricultural University

\section{Wenchao Li}

Hunan Agricultural University

\section{Xinrui Xie}

Hunan Agricultural University

\section{Yisong Liu}

Hunan Agricultural University

\section{Xi Zhang \\ Hunan Agricultural University \\ Wei Liu ( $\square$ weiliuprof@hunau.net ) \\ Hunan Agricutural University}

\section{Research Article}

Keywords: Spirometra tapeworm, sparganum, genetic diversity, multiplex PCR, snake

Posted Date: July 7th, 2021

DOI: https://doi.org/10.21203/rs.3.rs-648254/v1

License: (c) (i) This work is licensed under a Creative Commons Attribution 4.0 International License. Read Full License 


\section{Abstract}

Background: Sparganosis, caused by the larval plerocercoid of Spirometra tapeworms, still poses a potential public health hazard in China. Snakes are an important source of infection for human sparganosis; however, insufficient studies on the population genetics of Spirometra isolates from snakes have occurred to date. This study collected 67 Spirometra isolates from snakes, and several definitive hosts (Panthera tigris, Prionailurus bengalensis, Felis silvestris), in 15 geographical locations in Hunan Province of central China. They were identified using a multiplex polymerase chain reaction assay. Next, these isolates' population genetic diversity and phylogenetic pattern were explored based on mitochondrial cytb and cox 1 genes.

Results: All Spirometra isolates collected in the Hunan Province belonged to the genotype of the Korean "Spirometra decipiens" (KJ599679). The genetic diversity analysis based on concatenated sequences revealed no distinct genetic structure among Spirometra populations.

Conclusions: The phylogenetic analysis supported the distinction between Polish and Chinese Spirometra isolates. The taxonomy of Spirometra spp. remains ambiguous; more morphological and molecular studies are necessary to clarify the systematics of the genus of Spirometra.

\section{Background}

The plerocercoid larva (sparganum) of Spirometra tapeworms can parasitize humans and represent an important foodborne parasitic zoonosis [1]. The Spirometra erinaceieuropaei plerocercoid is the main etiological agent of human sparganosis and may result in serious disease, including blindness, paralysis, and even death [2]. Human sparganosis occur worldwide, mostly in eastern and south-eastern Asian countries, such as China, Korea, Japan, and Thailand [3, 4].

As the most common second intermediate hosts in the life cycle of $S$. erinaceieuropaei, snakes and frogs transmit sparganosis to humans in China $[5,6]$. Humans can be infected by consuming raw or undercooked snake/frog meat or using raw snake/frog flesh in traditional poultices [3]. Recently, eating snake and frog meat has become increasingly popular [5]. Such customs may facilitate human infection with spargana in China. The number of reported human cases has exceeded 1300 in China, but the actual number of infections may be far higher because many cases may not be recognized or reported [7]. Therefore, sparganosis may pose a serious threat to human health in China. As a result, knowledge regarding the genetic characteristics of sparganum populations is valuable for preventing and controlling sparganosis in humans.

Extensive genetic studies on spargana isolated from frogs in different regions in China have been performed [8, 9, 10, 11, 12, 13]. However, snakes are a very important source of human sparganosis, and data on the population genetics of Spirometra tapeworms from snakes remain scarce. Therefore, we set out to perform an exhaustive genetic diversity analysis of Spirometra tapeworms isolated from different snakes in different geographical locations. We used mitochondrial cytochrome $\mathrm{b}(c y t b)$ and cytochrome $c$ oxidase subunit I (cox1) genes that are verified as suitable markers for inferring genetic population differences of Spirometra tapeworms [11, 14].

Moreover, using mitochondrial genes, or even the complete mitochondrial genome, the sparganum isolates collected in different locations in China were classified as $S$. erinaceieuropaei $[6,8,11,15,16]$. This classification suggests that $S$. erinaceieuropaei might be the only etiologic agent of sparganosis in China. No other species of Spirometra as a source of human infection has been reported in the country. However, two Spirometra species, S. erinaceieuropaei and S. decipiens, have been identified as species that can infect humans in Korea through morphological and genetic methods [17]. In addition, several sparganum isolates collected from snakes (Dinodon rufozonatum and Agkistrodon saxatilis) in China were identified as $S$. decipiens based on the multiplex polymerase chain reaction (PCR) assay [18]. Therefore, the exact identification of Spirometra species from snakes requires further investigation. More specifically, the aims of this study were as follows: (1) to identify Spirometra isolates from snakes using multiplex PCR; (2) to perform a genetic diversity analysis of collected isolates from different geographical locations. 


\section{Materials And Methods \\ Collection of sparganum isolates from snakes}

Simples were collected from a field site in 15 geographical locations of the Hunan Province in southern China from April and September 2018 (Fig. 1, Supplementary Table S1). The presence of spargana was examined according to the methods of Liu [5]. In brief, snakes were euthanized using ethyl-ether anesthesia and skinned. We observed muscles and subcutaneous tissues with the naked eye, removed spargana with a picking needle, and placed them in phosphate-buffered saline to count them and observe their shapes and movements. Collected plerocercoids were fixed in $99 \%$ ethanol and kept at $-20^{\circ} \mathrm{C}$ for molecular analysis.

\section{Sequencing of target genes}

Total genomic DNA was extracted from individual plerocercoids using the Wizard $®$ SV Genomic DNA Purification System (Promega, Madison, Wisconsin, USA), following the manufacturers protocol. Two mitochondrial markers (cox 1 and cytb) were amplified by PCR using the primer combinations listed in Supplementary Table S2. PCR products were purified using the EasyPure PCR Purification Kit (Transgen, China) and sequenced in both directions at the Tsingke Company (Beijing, China).

\section{Multiplex PCR}

The multiplex PCR method, established by Jeon, identified the collected sparganum isolates [19]. In brief, two sets of speciesspecific primers, Se/Sd-1800F + Se-2018R plus Se/Sd-7955F + Se-8356R, and Se/Sd-1800F + Sd-2317R plus Se/Sd-7955F + Sd-8567R (Supplementary Table S3), were designed based on the mitochondrial nucleotide sequences of $c y t b, N A D H$ dehydrogenase subunit 4L (nad4L), NADH dehydrogenase subunit 4 (nad4), cox1, and large subunit ribosomal DNA (IrDNA) from S. erinaceieuropaei (KJ599680) and S. decipiens (KJ599679). As described by Jeon, using a combination of $S$. erinaceieuropaei-specific primers (Se/Sd-1800F + Se-2018R; Se/Sd-7955F + Se-8356R) would generate two S. erinaceieuropaei-specific bands of 239 base pairs (bp) and $401 \mathrm{bp}$, respectively [19]. In contrast, the S. decipiens-specific primers (Se/Sd-1800F + Sd-2317R; Se/Sd-7955F + Sd-8567R) would generate two S. decipiens-specific bands of 540 bp and $644 \mathrm{bp}$, respectively.

\section{Genetic diversity analysis of S. erinaceieuropaei isolates from snakes}

The DNA sequences for cytb and cox 1 were initially aligned using the default settings in the program Clustal X v.2.0 and adjusted in MEGA v.7.0 according to their amino acid sequences [20,21]. The nucleotide composition, conserved sites, variable sites, parsimony-informative sites, and singleton sites were estimated using MEGA v.7.0. We inferred the haplotypes of $S$. erinaceieuropaei isolates in DnaSP v. 6 based on cytb and cox 1 and used the same program to calculate values of genetic diversity per population [22]. The median-joining network presenting the relationship among all obtained haplotypes was prepared using PopART v.1.7 [23]. Analysis of molecular variance was computed using Arlequin v.3.5.1 to detect the partitions of genetic diversity within and among populations [24]. Pairwise genetic distances were also estimated using Arlequin v.3.5.1 to explore levels of genetic differentiation among the populations. To test for the demographic change to the S. erinaceieuropaei population, we performed neutrality tests using Arlequin v.3.5.1 with mismatch distribution using sum of squared deviations and raggedness index (RI) between observed and expected mismatches, as well as Fu's $F_{\mathrm{S}}$ test and Tajima's $D[25,26]$. To estimate the change in population size over time, we implemented the Bayesian skyline plot analysis in BEAST v2.5.2 [27]. Two independent analyses were performed with the concatenated sequences. We selected a piecewiseconstant skyline model using a relaxed, uncorrelated, log-normal molecular clock. Second, the molecular evolutionary rates for cytb and cox1 were selected as described by Kołodziej-Sobocińska [14].

\section{Phylogenetic analysis}

To perform a worldwide phylogenetic analysis of Spirometra tapeworms, we included all available complete sequences of cox 1 and cytb in GenBank in this study. We collected 181 concatenated sequences representing isolates from 36 
geographical locations in China and Poland (Supplementary Table S4). Four Diphyllobothrium species; Diphyllobothrium nihonkaiense (Genbank accession number representing the $c y t b / c o x 1$ genes: $A B 508837 / A B 015755), D$. latum (AB522608/AB511963), D. dendriticum (AB522613/KC812045), and D. ditremum (AB522617/FM209182) were used as the outgroup. The phylogeny of all collected sequences was estimated by the maximum likelihood (ML) and maximum parsimony (MP) methods. ML analysis was performed in PhyML v.3.0 using models selected by jModelTest 2 under the Akaike information criterion $[28,29]$. The support of each internal branch of the phylogeny was estimated using nonparametric bootstrapping (1,000 replicates). MP analyses were performed in PAUP*4b10 using heuristic searches with TBR branch swapping and 10,000 random addition sequences [30]. The confidence of each node was assessed by bootstrapping (2,000 pseudo-replicates, heuristic search of 20 random addition replicates with TBR option).

\section{Results And Discussion}

\section{Multiplex PCR-based identification}

We collected 55 sparganum isolates from two snake species (Zaocys dhumnades and Elaphe carinata) in 14 geographical locations in the Hunan Province of China. We also included 6 adult isolates from the tiger (Panthera tigris), 3 adult isolates from leopard cats (Prionailurus bengalensis), and 3 adult isolates from domestic cats (Felis silvestris) in one geographical location. All collected isolates were identified by multiplex PCR. In the multiplex PCR assay, when using the $S$. decipiensspecific primers (Se/Sd-1800F + Sd-2317R; Se/Sd-7955F + Sd-8567R), all collected isolates produced two S. decipiensspecific bands (540 bp and 644 bp) (Fig. 2). In contrast, when using the S. erinaceieuropaei-specific primers (Se/Sd-1800F + Se-2018R; Se/Sd-7955F + Se-8356R), no bands were observed. Therefore, according to the results of the multiplex PCR assay, all isolates collected in this study belonged to $S$. decipiens.

As described by Jeon, the specific primers for distinguishing $S$. erinaceieuropaei and $S$. decipiens were designed based on the mitochondrial genome sequences of $S$. erinaceieuropaei (KJ599680) and S. decipiens (KJ599679), respectively [19, 31]. However, the taxonomic position of S. erinaceieuropaei (KJ599680) and S. decipiens (KJ599679) remains controversial. Almeida found that $S$. erinaceieuropaei (KJ599680) from Korea differs significantly from other Asian isolates of the same species, suggesting that it is a different species of Spirometra [32]. Moreover, S. decipiens (KJ599679) is likely conspecific with the Asian isolates of $S$. erinaceieuropaei. After performing the comparative genetic analysis, Zhang also suggested that S. decipiens (KJ599679) should be a synonym of Asian S. erinaceieuropaei [6]. However, the Korean S. erinaceieuropaei (KJ599680) might be a specific genotype of $S$. erinaceieuropaei that differs from isolates from China and Japan. Yamasaki speculated that $S$. decipiens (KJ599679) is probably conspecific with Spirometra mansoni, and S. erinaceieuropaei (KJ599680) may represent an undescribed species [33]. In the most recent review, Kuchta concluded that $S$. decipiens (KJ599679) is synonymous with S. mansoni, and S. erinaceieuropaei (KJ599680) might represent a new species of Spirometra [2]. Given these observations and deductions, the exact taxonomic position of S. erinaceieuropaei (KJ599680) remains ambiguous. However, Yamasaki and Kuchta found support for considering S. decipiens (KJ599679) as synonymous with $S$. mansoni, and considering $S$. mansoni as a synonym of $S$. erinaceieuropaei $[2,33,34,35]$. To this end, most of the sequences submitted to GenBank were named $S$. erinaceieuropaei, and so the validation of the species $S$. mansoni remains to be verified $[13,33]$.

\section{Genetic diversity}

All amplifications for 67 Spirometra individuals were successful, with 1110-bp PCR products for cytb, and 1566-bp products for $\operatorname{cox} 1$. No insertions or deletions were found. We detected 119 polymorphic sites ( 61 for $c y t b$ and 58 for $\operatorname{cox} 1$ ), with 83 parsimony-informative sites ( 39 for $c y t b$ and 44 for $\operatorname{cox} 1$ ) and 36 singleton sites ( 22 for $c y t b$ and 14 for $\operatorname{cox} 1$ ). The concatenated sequences identified 48 haplotypes within the 67 isolates, which originated from 15 localities. Both individual and combined sequences for cox 1 and cytb had high $\mathrm{Hd}$, accompanied by low Pi (Table 1), which was in agreement with previous analysis of Spirometra isolates from frogs from different locations of China and isolates from different hosts in Poland $[10,14]$. 
Table 1

Genetic diversity indices of cox 1 and cytb genes in Spirometra mansoni from Hunan Province of China, including sampling size (SS), number (n) of haplotypes, haplotype diversity (Hd) and nucleotide diversity (Pi).

\begin{tabular}{|lllll|}
\hline mtDNA gene & $\mathrm{SS}$ & $\mathrm{n}$ haplotypes & $\mathrm{Hd}$ & $\mathrm{Pi}$ \\
\hline cox 1 & 67 & 30 & 0.899 & 0.0105 \\
\hline cytb & 67 & 26 & 0.888 & 0.0117 \\
\hline cox $1+$ cytb & 67 & 48 & 0.979 & 0.0110 \\
\hline
\end{tabular}

Analysis of molecular variance indicated that most of the observed genetic variation occurred within the 14 endemic populations $(70.57 \%)$, whereas the difference among the populations contributed $24.96 \%$ to the total population (Supplementary Table S5). The pairwise fixation index $\left(F_{\mathrm{ST}}\right)$ values between specified regions were estimated to measure the population differentiation (Table 2). Across all estimated 91 pairwise $F_{\mathrm{ST}}$ values, only 22 exhibited statistical significance. Within these 22 statistically significant $F_{\mathrm{ST}}$ values, most $F_{\mathrm{ST}}$ values between $\mathrm{CS}$ and other endemic regions were above 0.25 , such as that between CS and YiY, CS and LD, CS and CZ, CS and HH, CS and YuY, CS and XX. These findings indicated high genetic differences between isolates from CS and isolates from other geographical regions in the Hunan Province [36]. One reason for this observation might be that samples from CS were isolated from four different host species (Zaocys dhumnades, Panthera tigris, Prionailurus bengalensis, and Felis silvestris). 
Table 2

Estimates of pairwise $F_{\mathrm{ST}}$ of concatenate sequences between Spirometra populations.

\begin{tabular}{|c|c|c|c|c|c|c|c|c|c|c|c|c|c|c|}
\hline Sample & 1 & 2 & 3 & 4 & 5 & 6 & 7 & 8 & 9 & 10 & 11 & 12 & 13 & 14 \\
\hline $1 \mathrm{YiY}$ & 0.00 & & & & & & & & & & & & & \\
\hline $2 \mathrm{CD}$ & -0.19 & 0.00 & & & & & & & & & & & & \\
\hline $3 Y Z$ & -0.04 & -0.03 & 0.00 & & & & & & & & & & & \\
\hline $4 \mathrm{HY}$ & 0.24 & $\begin{array}{l}0.27 \\
\star\end{array}$ & $\begin{array}{l}0.29 \\
*\end{array}$ & 0.00 & & & & & & & & & & \\
\hline $5 \times \mathrm{T}$ & $\underset{*}{0.17}$ & 0.17 & -0.03 & 0.20 & 0.00 & & & & & & & & & \\
\hline $6 S Y$ & 0.32 & 0.35 & 0.28 & -0.00 & 0.14 & 0.00 & & & & & & & & \\
\hline $7 \mathrm{ZZ}$ & $\underset{*}{0.43}$ & $\underset{*}{0.47}$ & $\underset{*}{0.43}$ & 0.07 & $\underset{*}{0.32}$ & -0.17 & 0.00 & & & & & & & \\
\hline $8 \mathrm{CS}$ & $\begin{array}{l}0.37 \\
*\end{array}$ & 0.39 & 0.35 & 0.11 & 0.21 & -0.13 & $\begin{array}{l}-0.07 \\
*\end{array}$ & 0.00 & & & & & & \\
\hline 9 LD & 0.16 & 0.27 & 0.22 & 0.24 & 0.23 & 0.32 & 0.43 & 0.40 & 0.00 & & & & & \\
\hline $10 \mathrm{CZ}$ & -0.10 & -0.04 & -0.08 & 0.31 & 0.11 & 0.38 & 0.51 & 0.41 & 0.31 & 0.00 & & & & \\
\hline $11 \mathrm{HH}$ & -0.24 & -0.13 & 0.03 & 0.09 & 0.16 & 0.17 & 0.29 & $\begin{array}{l}0.29 \\
*\end{array}$ & 0.07 & 0.17 & 0.00 & & & \\
\hline $12 \mathrm{ZJJ}$ & $\begin{array}{l}0.30 \\
*\end{array}$ & 0.33 & 0.17 & -0.01 & -0.08 & -0.21 & -0.05 & -0.11 & 0.28 & 0.33 & 0.17 & 0.00 & & \\
\hline 13 YuY & 0.04 & 0.05 & 0.09 & 0.36 & 0.18 & 0.42 & 0.54 & $\begin{array}{l}0.44 \\
*\end{array}$ & $\underset{*}{0.26}$ & 0.11 & 0.10 & 0.40 & 0.00 & \\
\hline $14 X X$ & -0.04 & 0.02 & 0.00 & 0.30 & 0.12 & 0.36 & $\begin{array}{l}0.49 \\
\star\end{array}$ & $\underset{*}{0.41}$ & 0.27 & 0.12 & 0.04 & 0.31 & 0.03 & 0.00 \\
\hline
\end{tabular}

Analysis of the concatenated sequences showed no distinct genetic structure across the sampled Spirometra populations in the Hunan Province. In the median-joining network, all 67 sequences united in a star-like shape (Fig. 3). Although the haplotypes were high (48 haplotypes), no segregation by population, region, or host species was detected. Haplotype 26 was the most prominent haplotype and represented by eight samples from two regions (CS and ZZ) and isolated from all five host species. Neutrality tests of Tajima's $D$ and Fu's $F_{\mathrm{S}}$ for the whole Hunan dataset showed a significant negative value of Fu's $F_{\mathrm{S}}$ $\left(F_{S}=-7.7694, p=0.043\right)$ but a non-significant positive value of Tajima's $D(D=0.6439, p=0.798)$. Mismatch distribution analyses revealed multi-modal frequency distributions, which did not support a demographic expansion of Spirometra populations in the Hunan Province (Fig. 4). In addition, low values of the sum of squared deviation and RI under the demographic expansion model were found. The results of Bayesian skyline plot analyses also rejected sudden population expansion.

\section{Phylogenetic pattern}


We collected 67 sparganum isolates from 15 geographical locations of the Hunan Province. We explored the phylogenetic diversity of Spirometra isolates and compared the genetic variance between the isolates collected in frogs from other geographical locations of China and isolates from Poland (Supplementary Table S4) using the concatenated DNA sequences of cytb and cox1. Basal identical tree topologies were generated through phylogenetic inference based on both ML and MP methods. The phylogenetic pattern based on the MP analysis is demonstrated in Fig. 5. All collected isolates were segregated into two distinct clades (Clade 1 and Clade 2 ) with high support bootstrap values (bootstrap values $=100$ ). Clade 1 and Clade 2 comprised isolates from Poland and China, respectively. However, there was no obvious phylogenetic structure within each of the two clades. Among the Chinese isolates (Clade 2), although several isolates in snakes and isolates in frogs were grouped together to form some sub-clades, the support values of these sub-clades were too low to provide sufficient support for the phylogenetic patterns observed. This could indicate that the Chinese isolates should be considered a single genotype.

The distinct genetic separation of the Polish and Chinese populations of Spirometra isolates was identified by KołodziejSobocińska [14]. Our phylogenetic analysis supported the division of Polish and Chinese Spirometra isolates. Using a global full-length DNA sequence dataset of cox1, Kuchta performed a comprehensive phylogenetic analysis of Spirometra tapeworms [2]. They suggested there are at least six distinct lineages of the genus:

1. S. mansoni lineage (corresponding to most of the isolates from Asia, Australia, Romania, and a single sequence from Tanzania),

2. Spirometra sp. 1 lineage (corresponding to a few specimens from Korea and Japan),

3. S. folium lineage (corresponding to most specimens from Africa, such as Sudan, Ethiopia, and the remaining specimens from Tanzania),

4. S. erinaceieuropaei lineage (corresponding to the majority of the European specimens),

5. S. decipiens complex 1 lineage, and

6. S. decipiens complex 2 lineage.

Yamasaki re-examined Spirometra samples from Asia based on cox1 DNA sequence data and suggested two distinct Spirometra species to be present in Asi; Type I and Type II [33]. Type I is genetically diverse and widely distributed; however, Type II has only been found in Japan and Korea. Regarding Chinese Spirometra isolates, Zhang explored the genetic diversity of sparganum isolates in frogs from eastern, central, southern, and southwest China [10,11]. They found that the Chinese Spirometra population probably comprised two sub-groups; one group was made up of isolates mainly from eastern and central China. The other group included isolates mainly from southern and southwest China. However, they found no distinct genetic structure of cytb and cox 1 genes among Spirometra populations in snakes and other hosts from Hunan Province. One possible reason could be that the geographic distances between populations studied here were relatively small. Until now, definitive morphological criteria for distinguishing Spirometra species, including spargana and adult worms, have not been established [33]. Therefore, the taxonomy of Spirometra spp. and their phylogenetic relationships remain ambiguous, and more morphological and molecular studies are warranted to clarify the systematics of the genus.

\section{Conclusions}

The multiplex PCR assay using specific primers designed based on Spirometra mitochondrial genomes showed that all Spirometra isolates collected in snakes and other hosts (tiger, leopard cat, and domestic cat) in the Hunan Province of central China appeared to belong to the same genotype as the Korean "S. decipiens" (KJ599679). The genetic diversity analysis based on cytb and cox 1 genes revealed no distinct genetic structure among Spirometra populations in the Hunan Province. The phylogenetic analysis added support for the division of Polish and Chinese Spirometra isolates.

\section{Abbreviations}

cytb: mitochondrial cytochrome $b$ gene; cox1: cytochrome $c$ oxidase subunit I gene; PAUP: Phylogenetic Analysis Using Parsimony; TBR: tree-bisection reconnection; Hd: haplotype diversity; Pi: nucleotide diversity; HPD: highest probablity density. 


\section{Declarations}

\section{Ethics approval and consent to participate}

The Animal Ethics Committee approved all procedures involving animals of Hunan Agricultural University (43321503).

\section{Consent for publication}

Not applicable.

\section{Availability of data and materials}

All data generated or analyzed during this study are included in this published article and its additional files.

\section{Competing interests}

The authors declare that they have no competing interests.

\section{Funding}

Project support was provided by the Department of Science and Technology, Hunan Province (2016NK2014); the Department of Education, Hunan Province (17B126); the Natural Science Foundation of Henan Province of China (212300410070).

\section{Authors' contributions}

TG, XZ and WL designed the research study. TG, FFX, LF, JH, and XZ carried out the data acquisition, analysis, and interpretation. TG, FFX, and XZ wrote the manuscript. FL and WL edited the manuscript. All authors contributed to the conceptualization of the study and the revision of the manuscript.

\section{Author details}

${ }^{1}$ Research Center for Parasites \& Vectors, College of Veterinary Medicine, Hunan Agricultural University, Changsha, Hunan Province 410128, China; ${ }^{2}$ Department of Parasitology, School of Basic Medical Sciences, Zhengzhou University, Zhengzhou, Henan Province 450001, China; ${ }^{3}$ The Key Laboratory of Animal Vaccine \& Protein Engineering, Changsha, Hunan Province 410128, PR China.

\section{References}

1. Scholz T, Kuchta R, Brabec J. Broad tapeworms (Diphyllobothriidae), parasites of wildlife and humans: recent progress and future challenges. Int J Parasitol Parasites Wildl. 2019;9:359-69.

2. Kuchta R, Kołodziej-Sobocińska M, Brabec J, Młocicki D, Sałamatin R, Scholz T. Sparganosis (Spirometra) in Europe in the molecular era. Clin Infect Dis. 2021;72(5):882-90.

3. Liu Q, Li MW, Wang ZD, Zhao GH, Zhu XQ. Human sparganosis, a neglected food borne zoonosis. Lancet Infect Dis. 2015;15:1226-35.

4. Kim JG, Ahn CS, Sohn WM, Nawa Y, Kong Y. Human sparganosis in Korea. J Korean Med Sci. 2018;33:e273.

5. Liu W, Tan L, Huang Y, Li WC, Liu YS, Yang LC. Prevalence and molecular characterization of Spirometra erinaceieuropaei spargana in snakes in Hunan Province, China. J Helminthol. 2020;94:e131.

6. Zhang X, Hong X, Liu SN, Jiang P, Zhao SC, Sun CX, et al. Large-scale survey of a neglected agent of sparganosis Spirometra erinaceieuropaei (Cestoda: Diphyllobothriidae) in wild frogs in China. PLoS Negl Trop Dis. 2020;14(2):e0008019. 
7. Cui J, Wang Y, Zhang X, Lin XM, Zhang HW, Wang ZQ, et al. A neglected risk for sparganosis: eating live tadpoles in central China. Infect Dis Poverty. 2017;6(1):58.

8. Liu W, Zhao GH, Tan MY, Zeng DL, Wang KZ, Yuan ZG, et al. Survey of Spirometra erinaceieuropaei spargana infection in the frog Rana nigromaculata of the Hunan Province of China. Vet Parasitol. 2010;173:152-6.

9. Dai RS, Liu GH, Song HQ, Lin RQ, Yuan ZG, Li MW, et al. Sequence variability in two mitochondrial DNA regions and internal transcribed spacer among three cestodes infecting animals and humans from China. J Helminthol. 2012;86(2):245-51.

10. Zhang X, Wang H, Cui J, Jiang P, Fu GM, Zhong K, et al. Characterisation of the relationship between Spirometra erinaceieuropaei and Diphyllobothrium species using complete cytb and cox1 genes. Infect. Genet. Evol. 2015;35, 1-8.

11. Zhang X, Wang H, Cui J, Jiang P, Lin ML, Zhang YL, et al. The phylogenetic diversity of Spirometra erinaceieuropaei isolates from southwest China revealed by multi genes. Acta Trop. 2016;156:108-14.

12. Zhang X, Hong X, Duan JY, Han LL, Hong ZY, Jiang P, et al. Development of EST-derived microsatellite markers to investigate the population structure of sparganum - the causative agent of zoonotic sparganosis. Parasitology. 2019;146(7):947-55.

13. Hong X, Liu SN, Xu FF, Han LL, Jiang P, Wang ZQ, et al. Global genetic diversity of Spirometra tapeworms. Trop Biomed. 2020;37(1):237-50.

14. Kołodziej-Sobocińska M, Stojak J, Kondzior E, Ruczyńska I, Wójcik JM. Genetic diversity of two mitochondrial DNA genes in Spirometra erinaceieuropaei (Cestoda: Diphyllobothridae) from Poland. J Zool Syst Evol Res. 2019;57:764-77.

15. Tang TH, Wong SS, Lai CK, Poon RW, Chan HS. Molecular Identification of Spirometra erinaceieuropaei tapeworm in cases of human sparganosis, Hong Kong. Emerg Infect Dis. 2017;23:665-8.

16. Zhang X, Duan JY, Shi YL, Jiang P, Zeng DJ. Comparative mitochondrial genomics among Spirometra (Cestoda: Diphyllobothriidae) and the molecular phylogeny of related tapeworms. Mol Phylogenet Evol. 2017;117:75-82.

17. Jeon HK, Park H, Lee D, Choe S, Kim KH. Human Infections with Spirometra decipiens Plerocercoids Identified by Morphologic and Genetic Analyses in Korea. Korean J Parasitol. 2015;53:299-305.

18. Jeon HK, Park H, Lee D, Choe S, Kim KH. Genetic Identification of Spirometra decipiens Plerocercoids in Terrestrial Snakes from Korea and China. Korean J Parasitol. 2016;54:181-5.

19. Jeon HK, Kim KH, Sohn WM, Eom KS. Differential diagnosis of human sparganosis using multiplex PCR. Korean J Parasitol. 2018;56:295-300.

20. Larkin MA, Blackshields G, Brown NP, Chenna R, McGettigan PA, McWilliam H, et al. Clustal W and clustal X version 2.0. Bioinformatics. 2007;23:2947-8.

21. Kumar S, Stecher G, Tamura K. MEGA7: Molecular Evolutionary Genetics Analysis Version 7.0 for Bigger Datasets. Mol Biol Evol. 2016;33(7):1870-4.

22. Rozas J, Ferrer-Mata A, Sánchez-DelBarrio JC, Guirao-Rico S, Librado P, Ramos-Onsins SE, et al. DnaSP 6: DNA Sequence Polymorphism Analysis of Large Data Sets. Mol Biol Evol. 2017;34(12):3299-302.

23. Leigh JW, Bryant D. POPART: Full-feature software for haplotype network construction. Methods Ecol Evol. 2015;6:11106.

24. Excoffier L, Lischer HEL. Arlequin suite ver 3.5: a new series of programs to perform population genetics analyses under Linux and Windows. Mol Ecol Resour. 2010;10:564-7.

25. Fu YX. Statistical tests of neutrality of mutations against population growth, hitch-hiking, and background selection. Genetics. 1997;147:915-25.

26. Tajima F. Statistical method for testing the neutral mutation hypothesis by DNA polymorphism. Genetics. 1989;123:58595.

27. Bouckaert R, Heled J, Kühnert D, Vaughan T, Wu CH, Xie D, et al. BEAST 2: a software platform for Bayesian evolutionary analysis. PLoS Comput Biol. 2014;10(4):e1003537. 
28. Guindon S, Gascuel O. A simple, fast, and accurate algorithm to estimate large phylogenies by maximum likelihood. Syst Biol. 2003;52:696-704.

29. Darriba D, Taboada GL, Doallo R, Posada D. jModelTest 2: more models, new heuristics and parallel computing. Nat Methods. 2012;9:772.

30. Swofford DL. PAUP: Phylogenetic analysis using parsimony (and other metods). Version 4.0b.10. Sunderland: Sinauer Associates; 2013.

31. Eom KS, Park HS, Lee DM, Choe SJ, Kim KH, Jeon HK. Mitochondrial genome sequences of Spirometra erinaceieuropaei and S.decipiens (Cestoidea: Diphyllobothriidae). Korean J Parasitol. 2015; 53: 455-463.

32. Almeida GG, Coscarelli D, Melo MN, Melo AL, Pinto HA. Molecular identification of Spirometra spp. (Cestoda: Diphyllobothriidae) in some wild animals from Brazil. Parasitol Int. 2016;65(5 Pt A):428-31.

33. Yamasaki H, Sanpool O, Rodpai R, Sadaow L, Laummaunwai P, Un M, et al. Spirometra species from Asia: Genetic diversity and taxonomic challenges. Parasitol Int. 2021;80:102181.

34. Yamaguti S. The cestodes of vertebrates, Systema Helminthum. New York: II Interscience Publishers; 1959. pp. 358-61.

35. Iwata S. Experimental and morphological studies of Manson's tapeworm Diphyllobothrium erinacei (Rudolphi) special reference to its scientific name and relationship with Sparganum proliferum ljima, Prog. Med. Parasitol. Japan. Meguro Parasitological Museum, Tokyo. 1972; (4) pp. 533-90.

36. Balloux F, Lugon-Moulin N. The estimation of population differentiation with microsatellite markers. Mol Ecol. 2002;11(2):155-65.

\section{Figures}




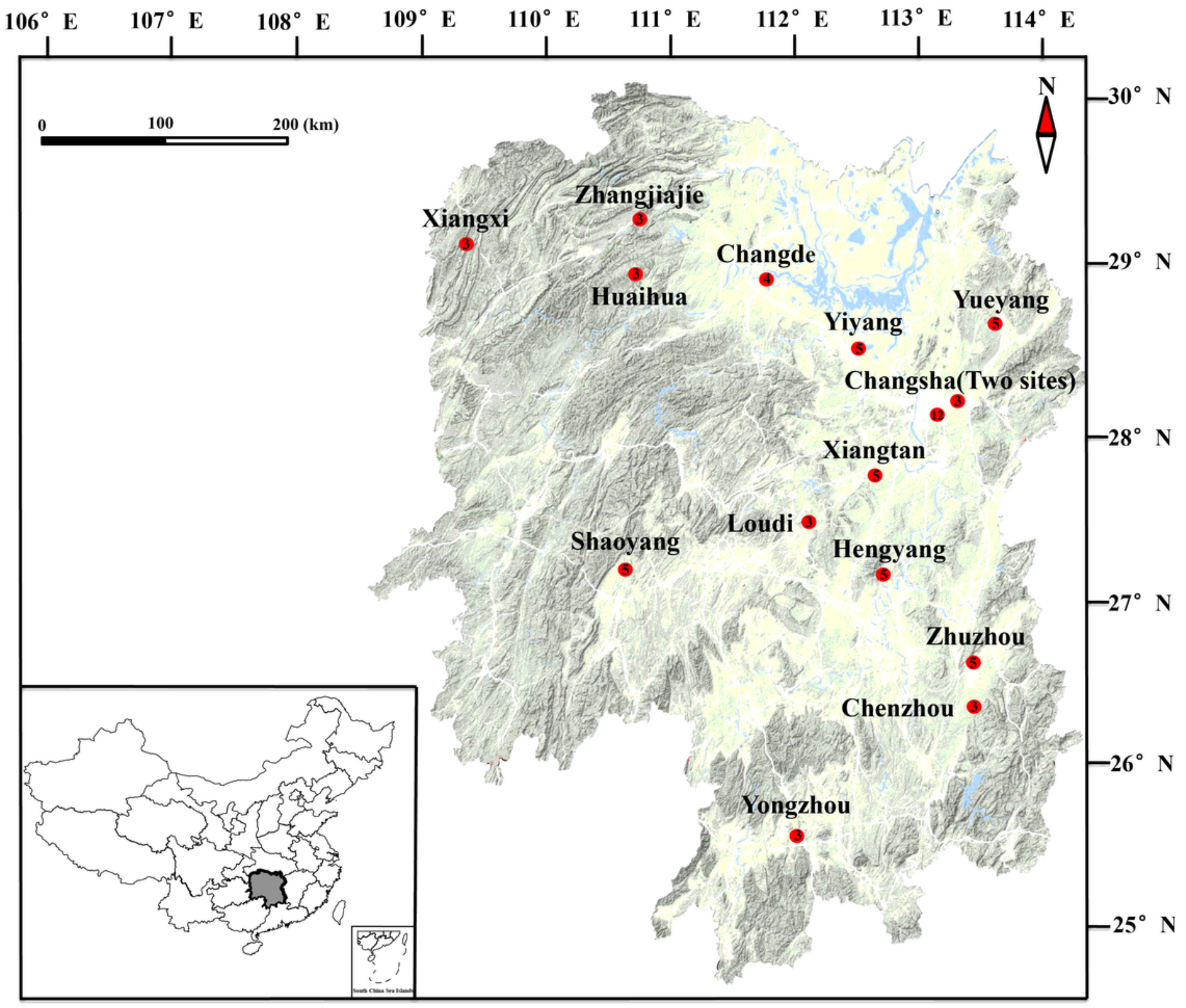

Figure 1

The sampling sites in Hunan Province, China. The geographic location was shown in the inset. The number on the red dot indicates the number of samples, and the sampling sites were added according to GPS record data. 


\section{$\begin{array}{llllllllllllllllll}M & 1 & 2 & 3 & 4 & 5 & 6 & 7 & 8 & 9 & 10 & 11 & 12 & 13 & 14 & \text { N1 } & \text { N2 } & \text { M }\end{array}$}

\section{0 bp \\ $1000 \mathrm{bp}$ \\ $750 \mathrm{bp}$ \\ 500 bp \\ $200 \mathrm{bp}$ \\ 100 bp}

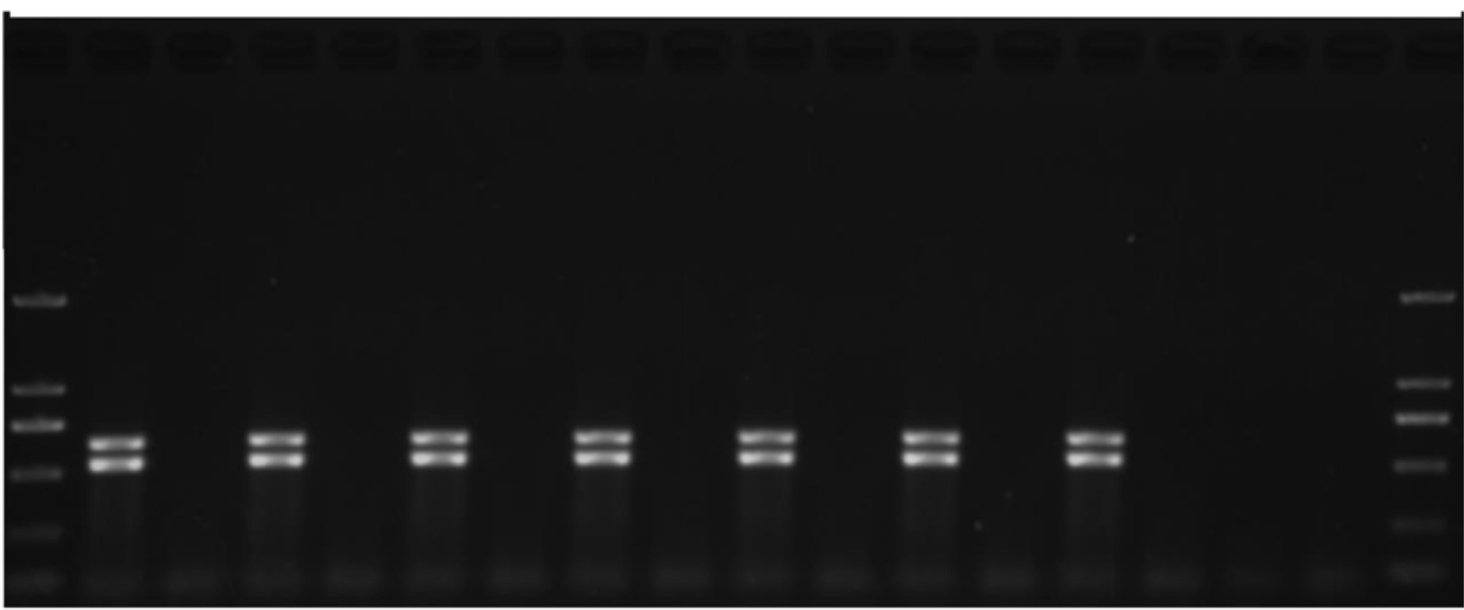

2000 bp

1000 bp

750 bp

500 bp

200 bp

$100 \mathrm{bp}$

Figure 2

An example of the multiplex PCR performed with species-specific primer sets. Lanes 1, 3, 5, 7, 9, 11, and 13 indicate PCR reactions with S. decipiens-specific primers (Se/Sd-1800F + Sd-2317R and Se/Sd-7955F + Sd-8567R). Lanes 2, 4, 6, 8, 10, 12 and 14 indicate PCRs with S. erinaceieuropaei-specific primers (Se/Sd-1800F + Se-2018R and Se/Sd-7955F + Se-8356R). Lanes 1 and 2, samples from Yiyang city; Lanes 3 and 4, samples from Changde city; Lanes 5 and 6, samples from Yongzhou city; Lanes 7 and 8, samples from Hengyang city; Lanes 9 and 10, samples from Xiangtan city; Lanes 11 and 12, samples from Shaoyang city; Lanes 13 and 14, samples from Zhuzhou city. M, DNA size marker (100 bp ladder). N1, negative control with S. decipiens-specific primers; N2, negative control with S. erinaceieuropaei -specific primers.

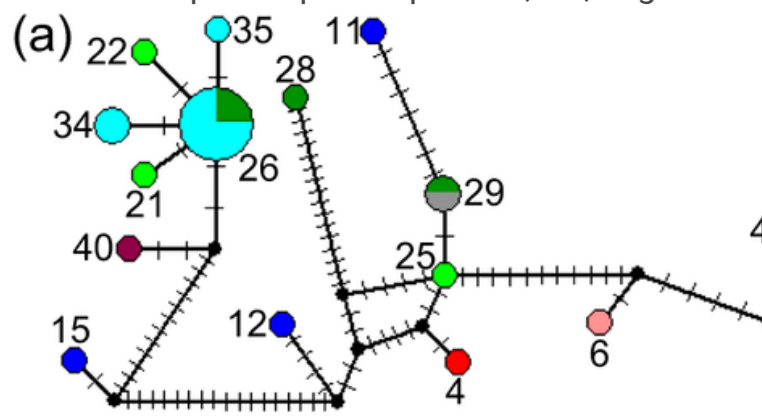

YiY City CD City YZ City $\mathrm{HY}$ City $\mathrm{XT}$ City SY City ZZ City CS City LD City XX City Cz City HH City ZJJ City YuY City

(b)

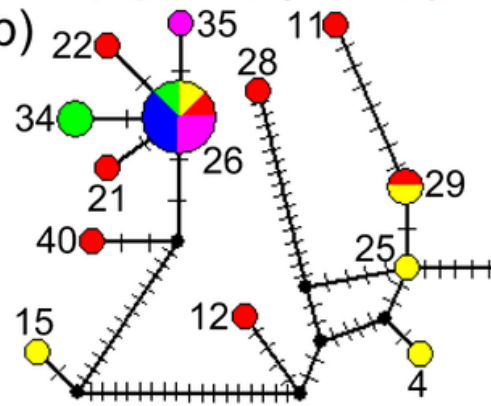

Zaocys dhumnades

Elaphe carinata

Panthera tigris

Prionailurus bengalensis

Felis silvestris
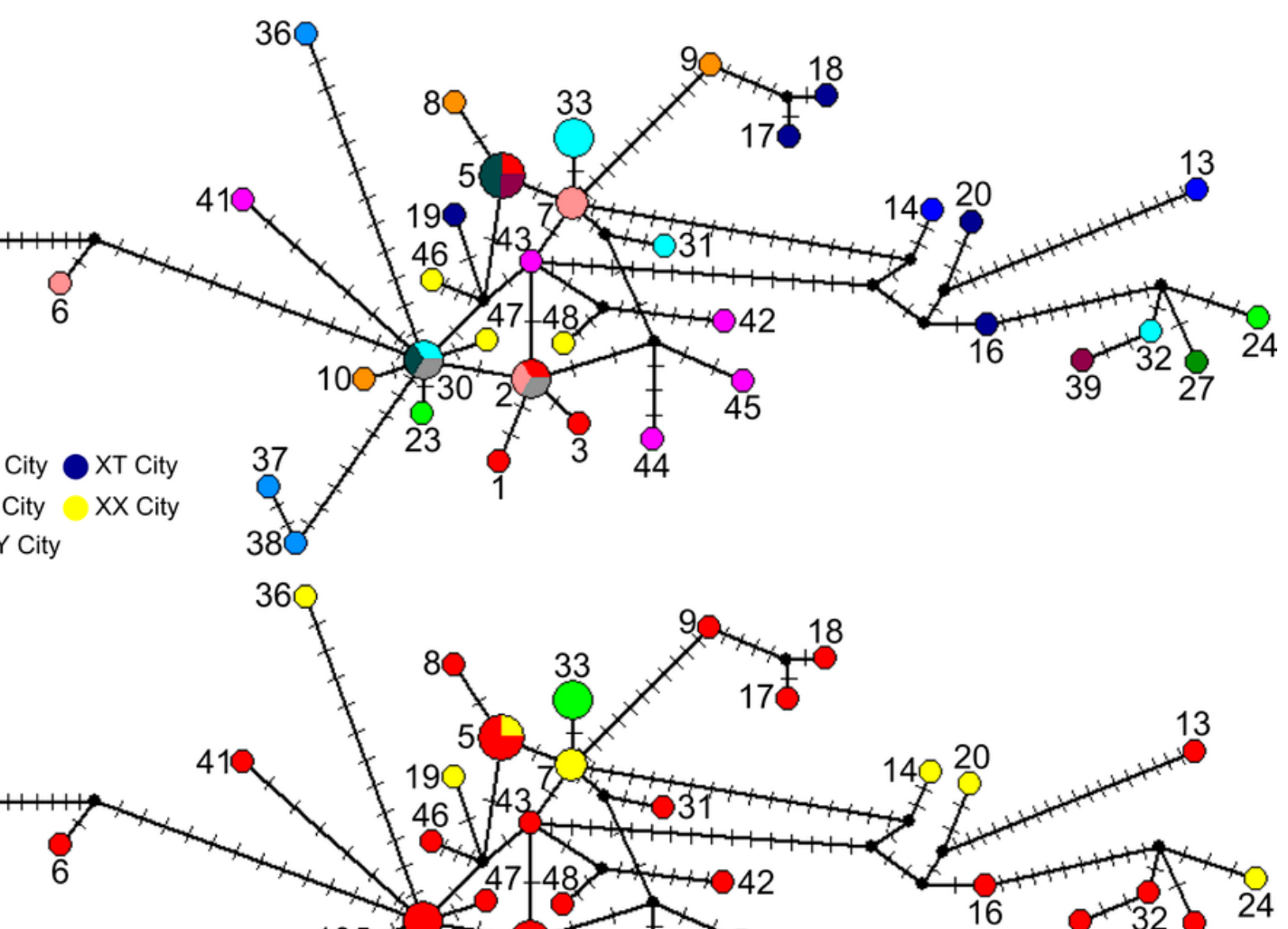


\section{Figure 3}

Median-joining network of 67 sequences of cytb and cox 1 genes in Spirometra isolates, colored by sampling sites (a) and host species (b) in the Hunan Province of China. The area of circles represents the number of individuals with that haplotype. Perpendicular short lines on the branches mark unsampled intermediate haplotypes.

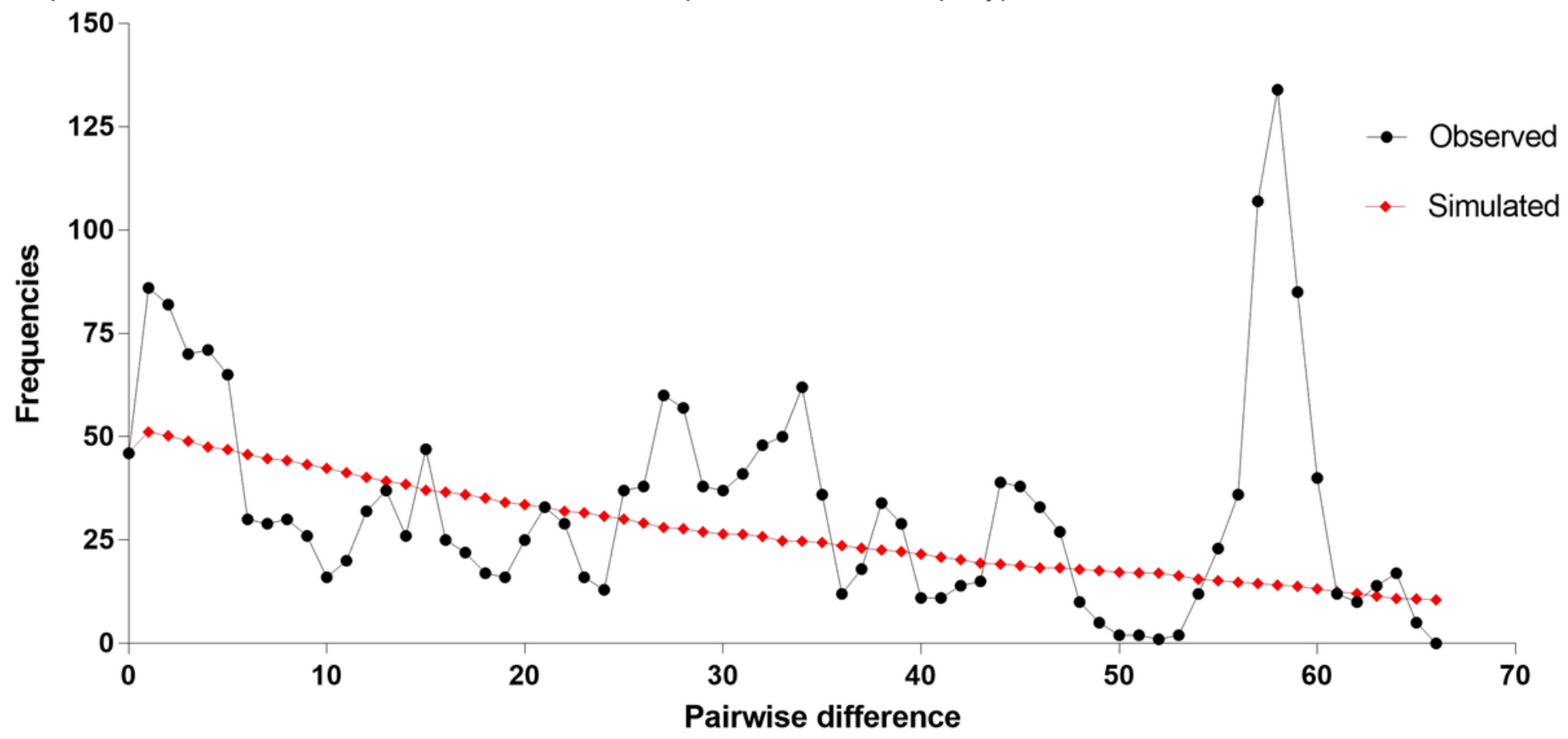

Figure 4

Mismatch distribution analyses. The line charts represent the observed frequencies of pairwise differences among haplotypes. 


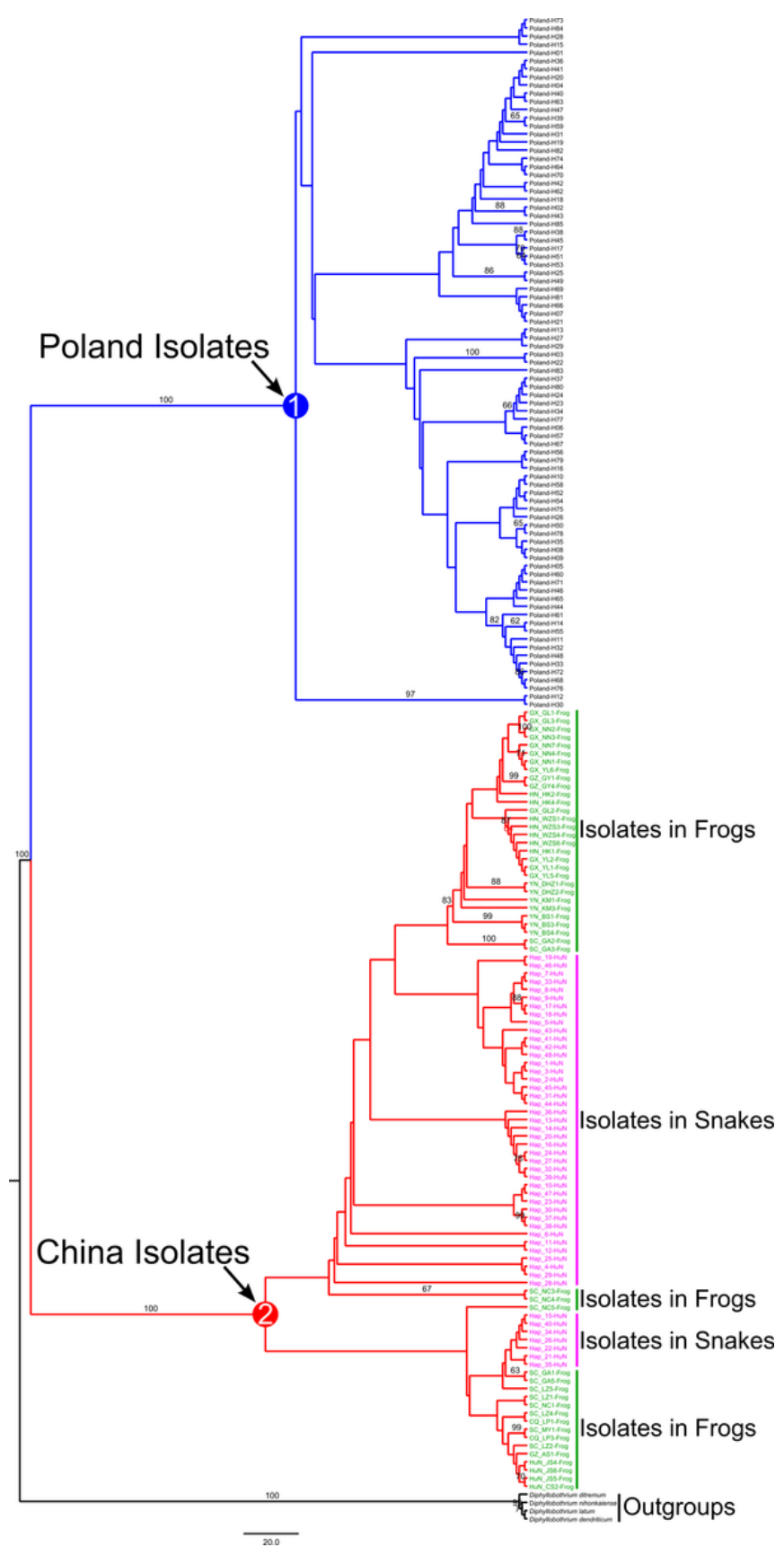

Figure 5

Phylogenetic relationships among the examined Spirometra isolates from different locations of China and Poland inferred by maximum parsimony (MP) analysis based on the concatenated DNA sequences of cytb and cox 1 . The numbers along the branches indicate bootstrap values, and bootstrap values higher than 60 are shown. Circled numbers represent the main clades discussed in the text.

\section{Supplementary Files}

This is a list of supplementary files associated with this preprint. Click to download.

- GraphicalAbstract.tif

- Tables1.doc

- Tables2.doc 
- TableS3.doc

- Tables4.doc

- Tables5.doc

Page 15/15 